\title{
Demystifying Discharge: Assessing Discharge Readiness to Predict Day of Discharge
}

\author{
Hemali Patel, MD*, Michelle Mourad, MD²
}

${ }^{1}$ Department of Medicine, Division of General Internal Medicine, Hospital Medicine Group, University of Colorado, Denver, Colorado; ${ }^{2}$ Department of Medicine, Division of Hospital Medicine, University of California San Francisco, San Francisco, California.

Widespread evidence suggests that the period around hospitalization remains a vulnerable time for patients. Nearly $20 \%$ of patients experience adverse events, including medication errors and hospital readmissions, within 3 weeks of discharge. ${ }^{1}$ Multiple factors contribute to adverse events, including the overwhelming volume of information patients receive on their last day in the hospital and fragmented interdisciplinary communication, both among hospital-based providers and with community providers. ${ }^{2-4}$ A growing body of literature suggests that to ensure patient understanding and a safe transition, discharge planning should start at time of admission. Yet, in the context of high patient volumes and competing priorities, clinicians often postpone discharge planning until they perceive a patient's discharge is imminent. "Discharge bundles," designed to improve the safety of hospital discharge, such as those developed by Project BOOST (Better Outcomes by Optimizing Safe Transitions) or Project RED (Re-Engineered Discharge), are not designed to help providers determine when a patient might be approaching discharge. ${ }^{5,6}$ Early identification of a patient's probable discharge date can provide vital information to inpatient and outpatient teams as they establish comprehensive discharge plans. Accurate discharge-date predictions allow for effective discharge planning, serving to reduce length of stay (LOS) and consequently improving patient satisfaction and patient safety. ${ }^{7}$ However, in the complex world of internal medicine, can clinicians accurately predict the timing of discharge?

A study by Sullivan and colleagues ${ }^{8}$ in this issue of the Journal of Hospital Medicine explores a physician's ability to predict hospital discharge. Trainees and attending physicians on general internal medicine wards were asked to predict whether each patient under their care would be discharged on the next day, on the same day, or neither. Discharge predictions were recorded at 3 time points: mornings (7-9 AM), midday (12-2 PM), or afternoons (5-7 PM). For predic-

*Address for correspondence and reprint requests: Hemali Patel, MD, 12401 E 17th Ave, Suite 450B, Mail Stop F-782, Aurora, CO 80045; Telephone: 720-848-4289; Fax: 720-848-4293; E-mail: hemali.patel@ucdenver.edu

Received: July 15, 2015; Accepted: July 24, 2015

2015 Society of Hospital Medicine DOI 10.1002/jhm.2445

Published online in Wiley Online Library (Wileyonlinelibrary.com). tions of next-day discharges, the sensitivity $(\mathrm{SN})$ and positive predictive value (PPV) were highest in the afternoon (SN 67\%, PPV 69\%), whereas for sameday discharges, accuracy was highest midday (SN $88 \%$, PPV $79 \%$ ). The authors note that physicians' ability to correctly predict discharges continually improved as time to actual discharge fell.

This study is novel; to our knowledge, no other studies have evaluated the accuracy with which physicians can predict the actual day of discharge. Although this study is particular to a trainee setting and more specific to a single academic medical center, the results are thought provoking. Why are attendings and trainees unable to predict next-day discharges more accurately? Can we do better? The majority of medical patients are not electively admitted and therefore may have complex and unpredictable courses compared to elective or surgical admissions. Subspecialty consultants may be guiding clinical care and potentially even determining readiness for discharge. Furthermore, the additional responsibilities of teaching and supervising trainees in academic medical centers may further delay discussions and decisions about patient discharges. Another plausible hypothesis, however, is that determination of barriers to discharge and discharge readiness is a "clinical skill" that is underappreciated and not taught or modeled sufficiently.

If we are to do better at predicting and planning for discharge, we need to build prompts for discharge readiness assessment into our daily work and education of trainees. Although interdisciplinary rounds are typically held in the morning, Wertheimer and colleagues show that additional afternoon interdisciplinary rounds can help identify patients who might be discharged before noon the next day. ${ }^{9}$ In their study, identifying such patients in advance improved the overall early discharge rate, moved the average discharge time to earlier in the day, and decreased the observed-to-expected LOS, all without any adverse effects on readmissions. We also need more communication between members of the physician care team, especially with subspecialists helping manage care. The authors describe moderate agreement with nextday and substantial agreement with same-day discharges between trainees and attendings. Although the authors do not reveal whether trainees or attendings were more accurate, the discrepancy with next-day 
discharges is notable. The disagreement suggests a lack of communication between team members about discharge barriers that can hinder planning efforts. Assessing a patient's readiness for and needs upon discharge, and anticipating a patient's disease trajectory, are important clinical skills. Trainees may lack clinical judgment and experience to accurately predict a patient's clinical evolution. As hospitalists, we can role model how to continuously assess patients' discharge needs throughout hospitalization by discussing discharge barriers during daily rounds. As part of transitions of care curricula, in addition to learning about best practices in discharge planning (eg, medication reconciliation, teach back, follow-up appointments, effective discharge summaries), trainees should be encouraged to conduct structured, daily assessment of discharge readiness and anticipated day of discharge.

Starting the discharge planning process earlier in an admission has the potential to create more thoughtful, efficient, and ultimately safer discharges for our patients. By building discharge readiness assessments into the daily workflow and education curricula, we can prompt trainees and attendings to communicate with interdisciplinary team members and address potential challenges that patients may face in managing their health after discharge. Adequately preparing patients for safe discharges has readmission implications. With Centers for Medicare and Medicaid Services reducing payments to facilities with high rates of readmissions, reducing avoidable readmissions is a priority for all institutions. ${ }^{10}$

We can accomplish safe and early discharges. However, we must get better at accurately assessing our patients' readiness for discharge if we are to take the first step.

Disclosure: Nothing to report.

\section{References}

1. Forster AJ, Murff HJ, Peterson JF, Gandhi TK, Bates DW. The incidence and severity of adverse events affecting patients after discharge from the hospital. Ann Intern Med. 2003;138(3):161-167.

2. Makaryus AN, Friedman EA. Patients' understanding of their treatment plans and diagnosis at discharge. Mayo Clin Proc. 2005;80(8): 991-994.

3. Dudas V, Bookwalter T, Kerr KM, Pantilat SZ. The impact of followup telephone calls to patients after hospitalization. Am J Med. 2001; 111(9 suppl 2):26-30.

4. Beers MH, Sliwkowski J, Brooks J. Compliance with medication orders among the elderly after hospital discharge. Hosp Formul. 1992; 27(7):720-724

5. Berkowitz RE, Fang Z, Helfand BKI, Jones RN, Schreiber R, PaascheOrlow MK. Project ReEngineered Discharge (RED) lowers hospital readmissions of patients discharged from a skilled nursing facility. J Am Med Dir Assoc. 2013;14(10):736-740.

6. Williams MV, Li J, Hansen LO, et al. Project BOOST implementation: lessons learned. South Med J. 2014;107(7):455-465.

7. Sumer T, Taylor DK, McDonald M, et al. The effect of anticipatory discharge orders on length of hospital stay in staff pediatric patients. Am J Med Qual. 1997;12(1):48-50.

8. Sullivan B, Ming D, Boggan JC, et al. An evaluation of physician predictions of discharge on a general medicine service. J Hosp Med. 2015;10(12):808-810.

9. Wertheimer B, Jacobs REA, Bailey M, et al. Discharge before noon: an achievable hospital goal. J Hosp Med. 2014;9(4):210-214.

10. Centers for Medicare \& Medicaid Services. Readmissions reduction program. Available at: http://cms.gov/medicare/medicare-fee-for-service-payment/acuteinpatientpps/readmissions-reduction-program.html. Updated August 4, 2014. Accessed July 10, 2015. 